\title{
Displasia Renal Asociada a Orificio Ureteral Ectópico en Vagina
}

\author{
Dra. M. Antonia Bidegaín S ${ }^{1}$; Dr. Eduardo Caffarena A. ${ }^{3}$; Dra. Sonia Figueroa Y.4; \\ Dr. Alfred Pugh M. ${ }^{2}$; Dr. Waldo Radríguez Y.4; Dra. Silvia Asenjo M. 5 . \\ Renal Dysplasia with Ectopic Vaginal Ureter
}

\begin{abstract}
Two cases of renal dysplasia associated with ectopic vaginal ureter are presented, both in females patients with urinary incontinence. In the first patient the whole left kidney was compromised by cystic renal dysplasia and drained in the vagina's left lateral wall. The second girl cystic renal dysplasia was limited to the upper left renal segment wich also drained through the left vaginal wall by means of a duplicated ureteral channel. Considering that vaginal structures derive from the Miiller's ducts the finding of vestigial Wolff's ducts remnants in $25 \%$ of normal women affords a plausible explanation to the ectopic ureteral drainage of this cases.

(Key words: Ectopic ureter. Renal dysplasia).
\end{abstract}

La coexistencia de displasia renal con anormalidades ipsilaterales de las vías urinarias ocurre, según Berstein, en el $90 \%$ de los casos.

A pesar de no haber una delinitación precisa entre las diversas formas de displasia, se distirguen una forma multiquística, una aplásica y otra hípoplásica. Las anomalías ureterales que presenta cada tipo son diferentes: las formas multiquística y aplásica se acompañan de ausencia de pelvis renal, atresia de uréter y a veces ausencia parcial o total del uréter. En el tipo hipoplásico puede existir estrechamiento ureteral, megauréter $y$, a veces ectopia ureteral con o sin ureterocele ${ }^{5}$.

La ectopia ureteral ocurre cuando el uréter desemboca en cualquier sitio que no sea el extremo pósterolateral del trígono. Es una anomalía poco frecuente, con franco predominio en el sexo femenino (6: 1). En alrededor de 70\% de los casos, el orificio ureteral ectópico se asocia con una duplicación ureteral que desemboca en la uretra 0 en los genitales accesorios. En la mujer, el orificio ectópico se abre habitualmente dentro de la zona de los esfínteres; pero cuando lo hace por fuera de ellos, en el trayecto del conducto de Gartner, se produce incontinencia

1. Servicio Pediatria, HospitalGuitlermo Grant Benavente.

2. Servicio Urología, Hospital Guillermo Grant Benavente.

3. Sección Urologia, Fac. de Medicina, Universidad de Concepción.

4. Depto. de Pediatría, Fac. de Medicina, Universidad de Concepción.

5. Residente Becaria Pediatría. urinaria permanente ${ }^{2}$. Esto no ocurre en el hombre, pues los tejidos del conducto de Wolf se extienden solamente hasta el verumontanum, por lo que la abertura no puede ser distal al esfínter externo. Según Kjellberg y colaboradores ${ }^{4}$, el orificio ureteral ectópico de las niñas se localiza en $38 \%$ de los casos en el vestíbulo; $32 \%$ en la uretra, $27 \%$ en la vagina y $3 \%$ en el útero. En cambio, en las series de la Clínica Mayo, los orificios ectópicos se describen con igual frecuencia en la porción periureteral del vestíbulo vaginal, la uretra distal y la región del cuello vesical ${ }^{3}$.

El propósito de esta comunicación es dar a conocer dos casos de incontinencia urinaria permanente en niñas portadoras de displasia renal asociada con desembocadura ureteral ectópica en la vagina.

\section{Casos Clínicos}

1. VMR, niña de 8 âtos de edad, con incontinencia urinaria permanente descubierta a la de edad de uno y medio años.

Tenía buen desarrollo pondo estatural, presión arterial normal, y normo tensión. En la región genital, se observaba emisión espontánea, lenta y permanente de orina a través del orificio himeneal. Los exámenes de sangre y orina eran normales; filtración glomerular de $90 \mathrm{ml} / \mathrm{min} /$ $1.73 \mathrm{~m}^{2}$. En la urografía excretora se encontró un sistema pielocaliciario rudimentario débilmente ${ }^{*}$ contrastado en el lado izquierdo (Fig. 1-A). En la Ecografía el riñón izquierdo era de tamaño reducido y estaba deformado. E1 Nefrograma 

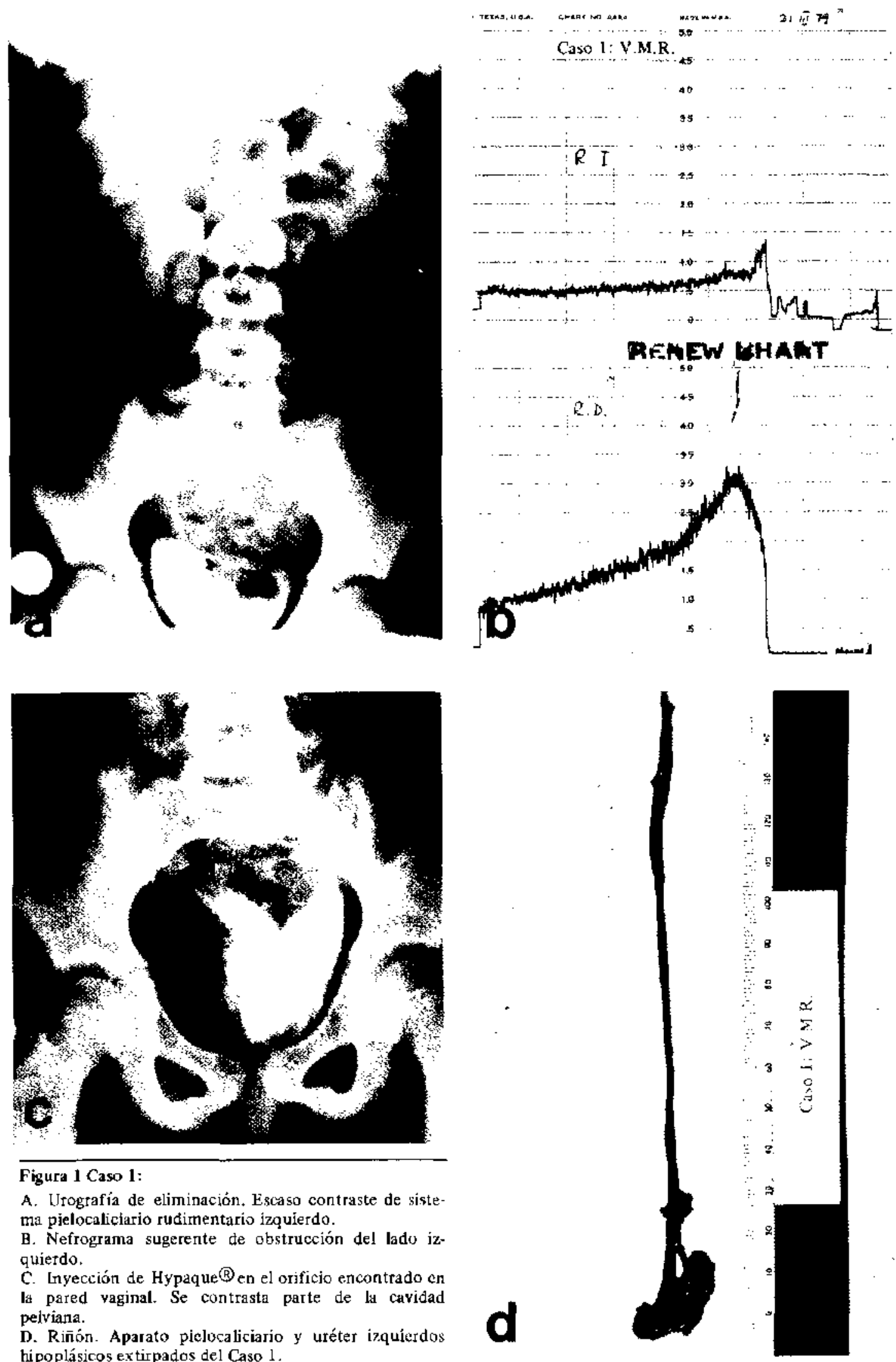

Figura 1 Caso 1:

A. Urografía de eliminación. Escaso contraste de sistema pielocaliciario ruđimentario izquierdo.

B. Nefrograma sugerente de obstrucción del lado izquierdo.

C. Inyección de Hypaque $($ en el orificio encontrado en la pared vaginal. Se contrasta parte de la cavidad pelviana.

D. Rin̄ón. Aparato pielocaliciario y uréter izquírdos hipoplásicos extirpados del Caso 1. 
registró en el Jado derecho una curva normal y en el izquierdo una curva sugerente de obstrucción (Fig. 1-B).

En la panendoscopia bajo anestesia general, se pudo observar que la vejiga tenía gran capacidad y la mucosa era normal; no se encontró orificio ureteral izquierdo mientras el derecho era normal. No se encontró fístula vésico-vaginal mediante el empleo de azul de metileno. En la pared izquierda de la vagina se observó, un orificio semejante al ureteral, el que, explorado con catéter e inyecciones de Hypaque ${ }^{\circledR}$, permitió contrastar una cavjdad, en la región media anterior de la pelvis, de contomos irregulares $y$ difusos, sin comunicación aparente con las asas intestinales y que correspondió a una extravasación del medio de contraste hacia la cavidad pelviana (Fig. $\mathbf{I}-\mathrm{C}$ ).

En la intervención quirúrgica se confirmó la existencia de hipoplasia renal izq̨uierda con orifj. cio ureteral ectópico en la vagina y se extirparon las estructuras anómalas (Fig. l-D).

La masa renal era irregular de $2,5 \times 1,5 \mathrm{~cm}$. De ella nacía un órgano tubular de $9 \times 0,2 \mathrm{~cm}$. Histológicamente la estructura renal formaba lóbulos, con septos fibrosos, vasos sanguj́neos y 3 a 4 quistes revestidos de epitelio cúbico; algunos lóbulos pequeños contenían sólo túbulos, otros, túbulos y cierta cantidad de glométulos. El uréter era bien constituido, su endotelio estaba plegado y rodeado de una gruesa capa muscular. (Displasia renal hipoplásića con hipoplasia ureteral). (Fig. 2).

La paciente ha evolucionado sin problemas y con función renal normal.

2. VCO, niña de 14 años, con incontinencia urinaria permanente. A la edad de 9 años se efectuó urografía excretora y citoscopía, que fueron interpretadas como normales, concluyéndose que tenia incontinencia de esfuerzo y se realizó una uretropexia. Dos meses después se comprobó que persistía la incontinencia pero la paciente abandonó los controles hasta recientemente por continuar con incontinencia urinaria permanente, además de sus micciones normales. En el examen gerital se comprobó emisión espontánea de orina por la vagina. Los exámenes hematológicos y de orina fueron normales. La urografia excretora mostró un doble sistema pieloureteral izquierdo, con el superior poco desarrollado y deformado. (Fig. 3-A). La uretro. cistografía fue normal. La panendoscopia simple dió resultados normales, pero después de administrar Clorhidrato de Fenazopiridina mostró salida de orina teñida por la pared izquierda de la vagina, no siendo posible cateterizar ol uréter ectópico.

Se practicó heminefrectomia superior y ureterectomia parcial izquierda. El segmento extirpado del riñón izquierdo tentáa múltiples quistes. Medía $4 \times 3 \times 2 \mathrm{~cm}$. En su superficie habían numerosos quistes transparentes de hasta $l \mathrm{~cm}$ de diámetro, alrededor de los cuales se veía tejido conectivo fibroso (Fig. 3-B). Microscópicamente el tejido renal estaba desorganizado y tenía quis. tes de diferentes tamaños revestidos por epitelio cúbico de una capa; a su alrededor había tejido fibroso que incluía túbulos aislados y glomérulos de cápsulas gruesas y asas lobulilladas (Displasia renal quística) (Fig. 3-C).

Evolucionó satisfactoriamente en el postoperatorio. La urografia excretora de control mostró buen contraste pielocalicilar, arquitectura conservada y buen contraste del uréter en el tercio inferior con desembocadura en la vejiga.

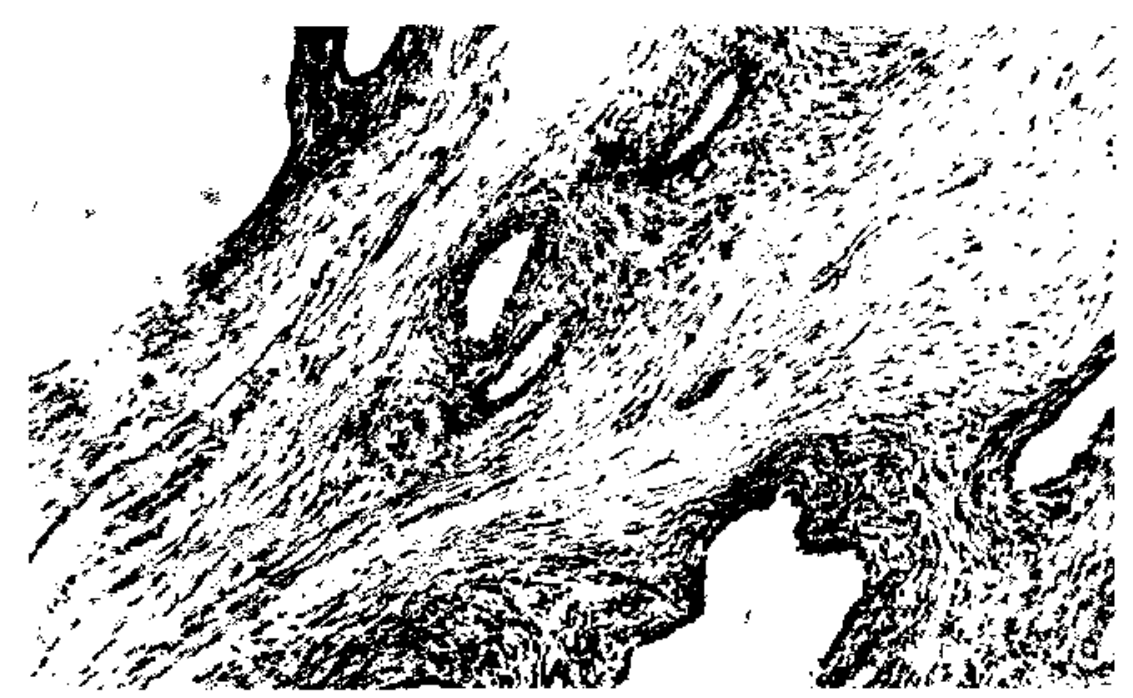

Figura 2. Aspecto histológico del tiñón en el Caso 1. 

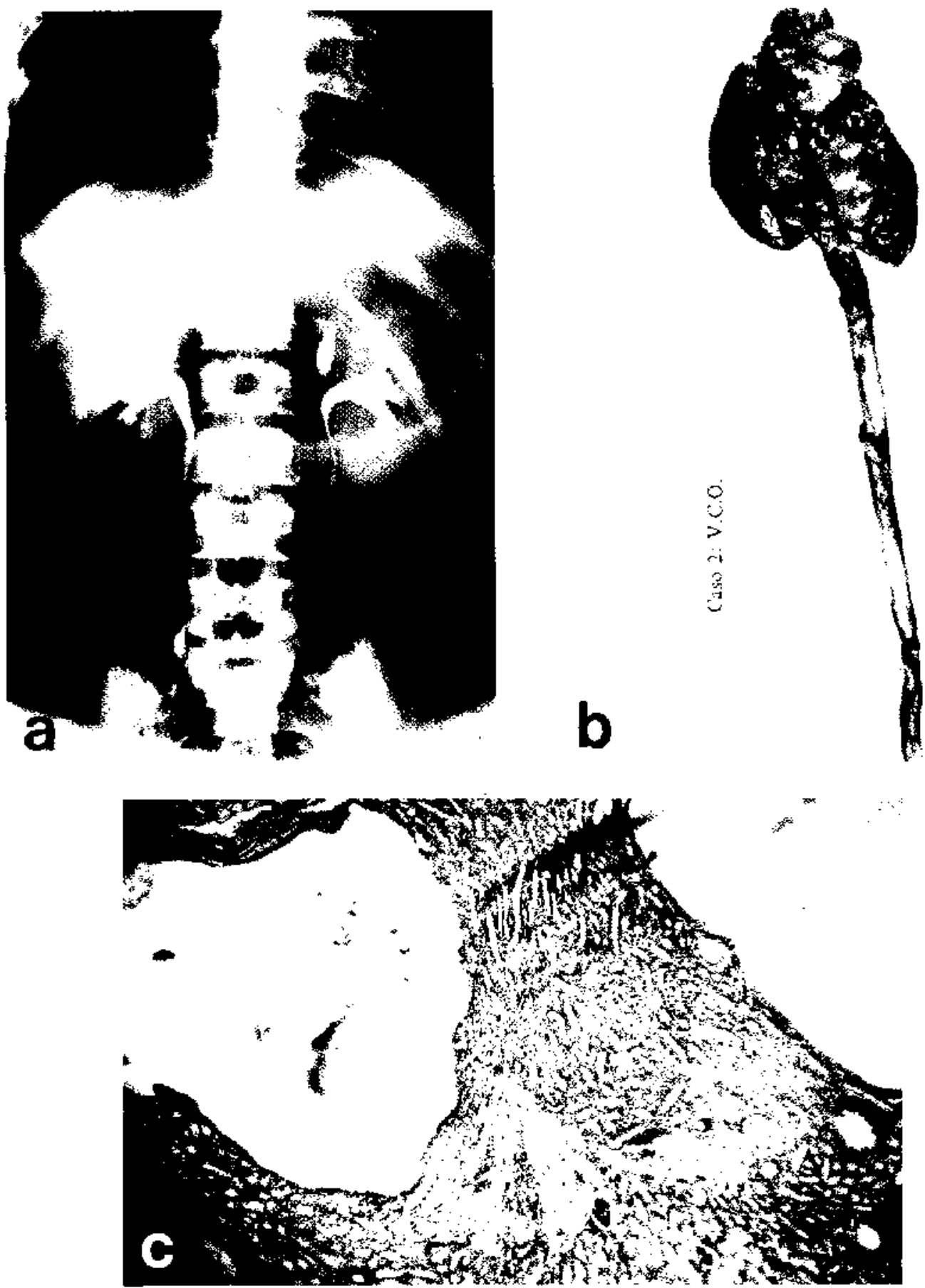

Figma 3: Caso 2.

A. Urograma excretor. Doble sistema pieloureteral izquierdo.

\section{COMENTARIO}

No hay aún una explicación satisfactoria para la existencia simultánea de displasia renal $y$ ectopía ureteral en la vagina.
B. Aspecto macrosiópico del segmento renal y el aparato piclo-ureteral 2 .

C. Aspecto microscópico del segmento extirpado del rin̄ón izquierdo.

La displasia renal es consecuencia de una diferenciación metanéfrica anómala. El proceso se reconoció histológicamente por desorganización del parénquima renal y por la presencia de estructuras nefrónicas y ductales inmaduras, 
anormalmente desarrolladas. E] término displasia renal fue en un comienzo aplicado sólo a riñones quísticos macroscópicamente malformados; pero en los últimos 20 años se ha extendido a casos con zonas focales de desarrollo anómalo, sólo detectables por examen mictoscópico, en riñones, que en otros aspectos están normalmente diferenciados.

Muchos rifrones congénitamente hipoplásicos también contienen zonas displásicas. La displasia renal abarca una amplia gama de presentaciones macroscópicas. Los riñones afectados pueden ser de mayor o menor tamatio que lo normal; total o parcialmente quísticos; de formas normales o alteradas ${ }^{4}$.

En la histólogía la displasia puede comprometer todo el rifión, o bien ser segmentaria of fucal.

La desembocadura ectópica del uréter en los sistemas únicos y dobles parece resultar de una falta de desarrollo de la porción terminal del conducto de Wolf (mesonefros) . La presencia de uréter ectópico en las estructuras derivadas del conducto de Wolf, como el cuello vesical, la uretra prostática, la vesícula seminal y el conduc. to deferente en el sexo masculino $y$ el cuello vesical, la uretra y el vestibulo en el sexo femenino, se explica por el origen común de estas estructuras. Sin embargo, es dificil explicar los orificios ectópicos en el útero, cérvix o vagina, pues estas estructuras derivan de los conductos de Müller. Se han encontrado remanentes de la porción distal del conducto de Wolf en la pared de la vagina, útero o ligamento ancho en $25 \%$ de las mujeres (Arey). La presencia de vestigios de estas estructuras sugicre una relación entre el conducto de Wolf, la vagina y el útero, que quizás puede of recer una explicación embriológica para la presencia de orificios ectópicos en dichas estructuras.

La histopatologia de los casos presentados corresponde a riñones displásicos asociađos con desembocadura ectópica del urèter en la vagina. Los riñones, aunque alterados, aún contienen parénquima renal normalmente diferenciado que permite cierta secreción de orina, lo que explica clinicamente la incontinencia urinaria permanen. te.

La combinación de ectopía ureteral única. desembocadura unilateral del uréter en la vagina en una mujer, en ausencia de ectopia renal. es extraordinariamente poco frecuente. Sólo sabe- mos 7 casos descritos en la literatura extranjera, en 4 el riñón afectado se situaba ectópicamente, fuera de los flancos.

En la segunda paciente, existía una ectopía ureteral doble, entidad también poco frecuente, pero mucho mejor documentada en la literatura. En su caso el uréter anómalo drenaba el sistema superior, que también era displásico. El riñón contralateral tenía un sistema pielo-ureteral normal; situación que también concuerda con 10 descrito.

La ectopía ureteral única parece ser un defec. to más precoz en la embriogenesis, por lo que se asociaria con mayor frecuencia a otras malformaciones congénitas, lo que no ocurrió en nuestra paciente.

Estas malformaciones, podrían ser pesquisadas thás oportunamente, si se examina en forma prolija los meatos urinarios y los genitales en los controles de salud de los niños, con o sin antece. dentes de incontinencia urinaria. Cuando existe evidencia de doble sistema pielo-ureteral, con alteración de la función o la morfología del hemiriłôn superior, es prudente realizar cistouretrografía miccional, panendoscopia, e incluso la pielografja retrógrada. El uso de colorantes como el clorhidrato de fenazopiridina y la vaginoscopía son de gran utilidad para localizar los orificios ureterales ectópicos.

\section{REFERENCIAS}

1. A mar A.: Lateral Uteteral Displacement, sign of non visualized duplication. J. Urol. 105: 638, $197 \mathrm{I}$.

2. Arey L.B.: Developmental Anatomy: A Textbook and Laboratory Manual of Hmbiology. Seventh ed. Philadelphia, W.B. Saunders Co., 295 314, 1974.

3. Kelalis P.P. and R. King.: Renal Pelvis and Ureter. Kelalis Panayotis P. Clinical Pediatric Urology Phila. delphia W.S. Saunders Company, 503-541, 1976.

4. Kjellberg S.E., Ericsonne, Rudhe U.: The Lower Urinary Tract in Childhood: Some correlated clinica! and Roentgenologic observations. Chicago year book medical publishers 1968.

5. Rudolph $A$, Hotfmat $J$ : Abnotmalitics of Renal Development. Bernstein $J$. and Moyer R. Pediatrics. Seventeenth Edition. Appleton-Century Crofts. $1175-1179,1982$.

6. Willams f:: Renal Agenesis, Dysplasia and Cistic Disease Paediatric Urology. Butterworth and $\mathrm{Co}$. Ltd. London $35 \quad 47,1976$.

7. Bernstein 1, : Developmental abnormalities of the renal parenchyma renal hypoplaxia and dysplasia Pathol. Annu $3 ; 213,1968$. 